\title{
ON GENERATORS AND PRESENTATIONS OF SEMIDIRECT PRODUCTS IN INVERSE SEMIGROUPS
}

\author{
E. R. DOMBI and N. RUŠKUC ${ }^{凶}$
}

(Received 6 March 2008)

\begin{abstract}
In this paper we prove two main results. The first is a necessary and sufficient condition for a semidirect product of a semilattice by a group to be finitely generated. The second result is a necessary and sufficient condition for such a semidirect product to be finitely presented.
\end{abstract}

2000 Mathematics subject classification: primary 20M05; secondary 20M18, 20M30.

Keywords and phrases: inverse semigroups, semidirect products, generators, presentations, groups, semilattices, actions.

\section{Introduction}

Investigation of finite generation and finite presentability of various constructions is one of the main areas of research in combinatorial semigroup theory. For example, finite generation and presentability of direct products of semigroups are considered in $[1,7]$. In [6] wreath products of monoids are discussed in this respect. Lavers gives a presentation for general products of monoids in [4] and investigates when general products of finitely presented monoids are finitely presented. In [3] finite generation and presentability of Schützenberger products are investigated. These constructions share the property that an action of one of the building blocks is defined on the other component. In the case of direct products, the action is trivial. A common feature of these results is that finite presentability of these constructions requires finite presentability of the component that is acted upon.

In inverse semigroup theory the construction of a semidirect product, which is an important example of general products, plays an important role. For example, every inverse semigroup divides a semidirect product of a semilattice by a group [5, Theorem 7.1.6]. Semilattices and groups are among the best-known examples of inverse semigroups. It is well known that a semilattice is finitely generated if and only if it is finite. The question naturally arises whether there are examples for finitely generated or finitely presented semidirect products of semilattices by groups where the

(C) 2009 Australian Mathematical Society 0004-9727/2009 \$16.00 
semilattice is infinite. As we shall see such examples do exist. In fact, finite generation or presentability relies on the new notion of finite generation or presentability with respect to the action.

Throughout the paper we are going to work with inverse semigroups. For the definition and basic properties of inverse semigroups see [5]. We briefly summarize basic facts regarding inverse semigroup presentations.

We consider inverse semigroups as algebras of type $(2,1)$, where the binary operation is multiplication and the unary operation assigns to each element its unique (von Neumann) inverse. First, we recall the description of the free inverse semigroup on a nonempty set $X$ as a factor semigroup of the free semigroup with involution on $X$; for more details the reader is referred to [5, Ch. 6].

Let $X$ be a nonempty set and $X^{-1}=\left\{x^{-1} \mid x \in X\right\}$. Consider the free semigroup $F=\left(X \cup X^{-1}\right)^{+}$and define a unary operation on $F$ in the following way: for each $y \in X \cup X^{-1}$, let

$$
y^{-1}= \begin{cases}x^{-1} & \text { if } y=x \in X, \\ x & \text { if } y=x^{-1} \in X^{-1},\end{cases}
$$

and define $\left(y_{1} \ldots y_{n}\right)^{-1}=y_{n}^{-1} \ldots y_{1}^{-1}$. Then $\left(F, \cdot,{ }^{-1}\right)$ is the free semigroup with involution on $X$, which we shall denote by $\operatorname{FSI}(X)$. Define the following binary relation on $F S I(X)$ :

$$
\Re=\left\{\left(u u^{-1} u, u\right) \mid u \in \operatorname{FSI}(X)\right\} \cup\left\{\left(u u^{-1} v v^{-1}, v v^{-1} u u^{-1}\right) \mid u, v \in \operatorname{FSI}(X)\right\} .
$$

The congruence generated by $\Re$ is called the Wagner congruence which we denote by $\rho$. The factor semigroup $F I(X)=F S I(X) / \rho$ is the free inverse semigroup on $X$. We refer to the elements of $\Re$ as standard inverse semigroup relations.

An inverse semigroup presentation is an ordered pair $\langle X \mid P\rangle$, where $P$ is a binary relation on $F S I(X)$. Let $\tau$ denote the congruence generated by $P \cup \Re$. The semigroup $S=F S I(X) / \tau$ is said to be presented as an inverse semigroup by the generators $X$ and relations $P$ and we denote this by $S=\operatorname{Inv}\langle X \mid P\rangle$.

Let $S=\operatorname{Inv}\langle X \mid P\rangle$ and let $w_{1}, w_{2}$ be words over $X \cup X^{-1}$. We write $w_{1} \equiv w_{2}$, if $w_{1}$ and $w_{2}$ are identical as words and we write $w_{1}=w_{2}$, if $w_{1}$ and $w_{2}$ represent the same element of $S$. If $w_{1}=w_{2}$, then we also say that $S$ satisfies the relation $w_{1}=w_{2}$. We say that $w_{2}$ is obtained from $w_{1}$ by an application of a relation of $P$ or of a standard inverse semigroup relation, if $w_{1} \equiv \alpha u \beta$ and $w_{2} \equiv \alpha v \beta$, where $\alpha, \beta \in\left(X \cup X^{-1}\right)^{*}$ and $u=v$ or $v=u$ is a relation of $P$ or a standard inverse semigroup relation. We say that $w_{1}=w_{2}$ is a consequence of relations in $P$ and of standard inverse semigroup relations, if there exists a sequence of words $w_{1} \equiv \alpha_{0}, \ldots, \alpha_{m} \equiv w_{2}$, where $\alpha_{j+1}$ is obtained from $\alpha_{j}$ by applying a relation of $P$ or a standard inverse semigroup relation. Usual general considerations give the following result.

Proposition 1.1. Let $S$ be an inverse semigroup generated by a set $X$. Then $S=\operatorname{Inv}\langle X \mid P\rangle$ if and only if the following two conditions hold:

(i) $S$ satisfies all relations in $P \cup \mathfrak{R}$;

(ii) if $w_{1}, w_{2} \in\left(X \cup X^{-1}\right)^{+}$are such that $w_{1}=w_{2}$ holds in $S$, then $w_{1}=w_{2}$ is a consequence of relations in $P \cup \mathfrak{R}$. 
One of the central notions of this paper is the notion of action. Let $(T, *)$ and $(S, \cdot)$ be semigroups. We say that $S$ acts on $T$ by endomorphisms (automorphisms) on the left, if for every $s \in S$ there exists an endomorphism (automorphism) $\phi_{s}: T \rightarrow T$ so that $\phi_{s_{1}} \phi_{s_{2}} t=\phi_{s_{1} s_{2}} t$ holds for all $s_{1}, s_{2} \in S, t \in T$. If $S$ is a monoid with identity 1 , then we also require $\phi_{1}: T \rightarrow T$ to be the identity map. For all $s \in S$ and $t \in T$ we denote $\phi_{s} t$ by ${ }^{s} t$. In this notation, the rule now becomes ${ }^{s_{1}}\left({ }^{s_{2}} t\right)={ }^{s_{1} s_{2}} t$.

Let $(T, *)$ and $(S, \cdot)$ be semigroups. Assume that $S$ acts on $T$ on the left by automorphisms. The semidirect product $T \rtimes S$ of $T$ by $S$ with respect to this action has as its underlying set $T \times S$ with multiplication defined by $(e, g)(f, h)=$ $\left(e *^{g} f, g h\right)$. This easily extends to

$$
\left(e_{1}, g_{1}\right) \ldots\left(e_{n}, g_{n}\right)=\left(e_{1} *^{g_{1}} e_{2} * \ldots *^{g_{1} \ldots g_{n-1}} e_{n}, g_{1} \ldots g_{n}\right) .
$$

It is well-known that if $T$ is a semilattice and $S$ is a group, then $T \rtimes S$ is an inverse semigroup (see for example [5, Theorem 7.1.1]).

\section{Generators}

We introduce the concept of finite generation of a semigroup with respect to a semigroup action. This notion will help us to give a necessary and sufficient condition for a semidirect product of a semilattice by a group to be finitely generated. Throughout the paper if we say that a group $G$ acts on a semilattice $Y$, then we mean that $G$ acts on $Y$ on the left by automorphisms.

Let $(Y, \wedge)$ be a semilattice and let $\leq$ denote the natural partial order on $Y$. Recall that $x \leq y$ if and only if $x=x \wedge y$. We say that $y \in Y$ is a maximal element of $Y$ if $x \in Y, y \leq x$ always implies that $x=y$. We say that $Y$ satisfies the maximum condition if it has finitely many maximal elements, and for every $x \in Y$ there exists a maximal element $y \in Y$ such that $x \leq y$. The following lemma is immediate from the definitions.

LEMMA 2.1. Let $(Y, \wedge)$ be a semilattice and let $\varphi$ be an automorphism of $(Y, \wedge)$. Then $x \leq y$ if and only if $x \varphi \leq y \varphi$. In particular, we have that $y$ is a maximal element of $Y$ if and only if $y \varphi$ is a maximal element of $Y$.

The following definition proves to be the key concept for the main result of this section.

Definition 2.2. Let $(S, \cdot)$ be a monoid and $(T, *)$ be a semigroup. Assume that $S$ acts on $T$ on the left by endomorphisms (automorphisms). We say that $T$ is generated by $T_{0} \subseteq T$ with respect to the action of $S$, if $T=\left\langle{ }^{S} T_{0}\right\rangle$, where ${ }^{S} T_{0}=\left\{{ }^{s} t \mid s \in S\right.$, $\left.t \in T_{0}\right\}$. We say that $T$ is finitely generated with respect to the action of $S$ if $T_{0}$ can be chosen to be a finite subset of $T$.

The following is the main result of this section. 
Proposition 2.3. Let $(Y, \wedge)$ be a semilattice, and $(G, \cdot)$ be a group acting on $Y$. The semidirect product $S=Y \rtimes G$ is finitely generated if and only if the following conditions hold:

(i) $G$ is finitely generated;

(ii) $Y$ satisfies the maximum condition;

(iii) $Y$ is finitely generated with respect to the action of $G$.

ProOF. ( $\Longrightarrow$ ) Assume that $S=Y \rtimes G$ is finitely generated by the elements $A=\left\{\left(e_{1}, g_{1}\right), \ldots,\left(e_{n}, g_{n}\right)\right\}$, where $A \subseteq S$. Without loss of generality we may assume that $(e, g)^{-1}=\left(g^{-1} e, g^{-1}\right) \in A$ for all $(e, g) \in A$. Let $X=\left\{g_{1}, g_{2}, \ldots, g_{n}\right\}$ and $Y_{0}=\left\{e_{1}, \ldots, e_{n}\right\}$. Let $(e, h) \in S$. Write $(e, h)=\left(f_{1}, h_{1}\right)\left(f_{2}, h_{2}\right) \ldots\left(f_{k}, h_{k}\right)$ where $\left(f_{j}, h_{j}\right) \in A$ for all $1 \leq j \leq k$. Then, on the one hand $h=h_{1} \ldots h_{k}$, where $h_{j} \in X$ verifying that $G$ is generated by the finite set $X$; on the other hand, $e=$ $f_{1} \wedge{ }^{h_{1}} f_{2} \wedge \cdots \wedge{ }^{h_{1} \ldots h_{k-1}} f_{k}$, where $f_{1}, \ldots, f_{k} \in Y_{0}$. It follows that $Y=\left\langle{ }^{G} Y_{0}\right\rangle$. We also obtain that $e \leq f_{1}$. Hence, the maximal elements of $Y$ are the maximal elements of $Y_{0}$ and we deduce that $Y$ satisfies the maximum condition.

( $\Longleftarrow$ ) For the converse assume that $G$ is generated by the finite set $X$, that $Y$ satisfies the maximum condition and that $\left\langle{ }^{G} Y_{0}\right\rangle=Y$ for some finite set $Y_{0} \subseteq Y$. Let $Y_{m}$ denote the set of maximal elements of $Y$. Without loss of generality we may assume that $Y_{m} \subseteq Y_{0}$. We claim that

$$
A=\left\{(e, 1) \mid e \in Y_{0}\right\} \cup\left\{(e, g) \mid e \in Y_{m}, g \in X \cup X^{-1}\right\}
$$

generates $S$.

Let $(e, h) \in Y_{0} \times G$. Let $\tilde{e} \in Y_{m}$ be such that $e \leq \tilde{e}$ and suppose that $h=g_{1} g_{2} \ldots g_{k}$ where $g_{1}, g_{2}, \ldots, g_{k} \in X \cup X^{-1}$. By Lemma 2.1, there exist $f_{1}, f_{2}, \ldots, f_{k-1} \in Y_{m}$ such that ${ }^{g_{1}} f_{1}=\tilde{e}$ and ${ }^{g} f_{j}=f_{j-1}$ for all $2 \leq j \leq k-1$. It follows that ${ }^{g_{1} \ldots g_{j}} f_{j}=\tilde{e}$ for all $1 \leq j \leq k-1$ and we obtain

$$
\begin{aligned}
(e, 1)\left(\tilde{e}, g_{1}\right)\left(f_{1}, g_{2}\right) \ldots\left(f_{k-1}, g_{k}\right) & =\left(e \wedge \tilde{e} \wedge{ }^{g_{1}} f_{1} \wedge \cdots \wedge \wedge^{g_{1} g_{2} \ldots g_{k-1}} f_{k-1}, h\right) \\
& =(e \wedge \underbrace{\tilde{e} \wedge \tilde{e} \wedge \cdots \wedge \tilde{e}}_{k}, h)=(e \wedge \tilde{e}, h)=(e, h)
\end{aligned}
$$

verifying that elements of $Y_{0} \times G$ are generated by $A$.

Let $e \in Y$ and $h \in G$ be arbitrary. Since $\left\langle{ }^{G} Y_{0}\right\rangle=Y$, there exist $h_{1}, \ldots, h_{k} \in G$ and $f_{1}, \ldots, f_{k} \in Y_{0}$ such that $e={ }^{h_{1}} f_{1} \wedge \cdots \wedge{ }^{h_{k}} f_{k}$. Since $Y$ satisfies the maximum condition, there exists $\tilde{e} \in Y_{m}$ such that $e \leq \tilde{e}$, and so $e=\tilde{e} \wedge e$ holds. Then

$$
(e, h)=\left(\tilde{e}, h_{1}\right)\left(f_{1}, h_{1}^{-1} h_{2}\right) \ldots\left(f_{k-1}, h_{k-1}^{-1} h_{k}\right)\left(f_{k}, h_{k}^{-1}\right)(\tilde{e}, h) .
$$

By the above argument each component of the product can be written in terms of elements of $A$ and thus $S$ is finitely generated by $A$. 


\section{Presentations with respect to a semigroup action}

In this section we introduce the notion of finite presentability of an inverse semigroup with respect to a semigroup action. This will be the key concept in giving a necessary and sufficient condition for a semidirect product of a semilattice by a group to be finitely presented.

Let $S$ be a monoid and $T$ be an inverse semigroup on which $S$ acts. Assume that $T$ is generated by $T_{0}$ with respect to this action. Let $Q$ be a binary relation on $F S I\left({ }^{S} T_{0}\right)$ and let

$$
Q_{A}=\left\{{ }^{s} p={ }^{s} q \mid(p=q) \in Q \cup \Re, s \in S\right\} .
$$

If $T=\operatorname{Inv}\left\langle{ }^{S} T_{0} \mid Q_{A}\right\rangle$, then we say that $T$ is presented as an inverse semigroup with respect to the action of $S$ by the generators $T_{0}$ and relations $Q$ and we denote this by $T=\operatorname{InvAct}_{S}\left\langle T_{0} \mid Q\right\rangle$. If $T_{0}$ and $Q$ can be chosen to be finite sets, then we say that $T$ is finitely presented as an inverse semigroup with respect to the action of $S$. The following proposition is as expected and can be proved using standard techniques. For further details see [2].

Proposition 3.1. Let $S$ be a monoid and $T$ be an inverse semigroup. Assume that $S$ acts on $T$. Assume that the finite sets $Y_{1}$ and $Y_{2}$ generate $T$ with respect to the action of $S$ and that $T$ can be defined by a finite inverse semigroup presentation with respect to the action of $S$ in terms of $Y_{1}$. Then $T$ can be defined by a finite inverse semigroup presentation with respect to the action of $S$ in terms of $Y_{2}$ as well.

The following is the main theorem of the paper.

THEOREM 3.2. Let $(Y, \wedge)$ be a semilattice and let $G$ be a group acting on $Y$ on the left by automorphisms. The semidirect product $S=Y \rtimes G$ is finitely presented as an inverse semigroup if and only if the following conditions hold:

(i) $G$ is finitely presented;

(ii) $Y$ satisfies the maximum condition;

(iii) $Y$ is finitely presented as an inverse semigroup with respect to the action of $G$.

Theorem 3.2 will be proved in the following two sections. We first make some preliminary observations and introduce the notation we use.

Let $Y$ be a semilattice and $G$ be a group acting on $Y$. Assume that the semidirect product $S=Y \rtimes G$ is generated as an inverse semigroup by the finite set $A$. For a word

$$
w \equiv\left(e_{1}, g_{1}\right) \ldots\left(e_{k}, g_{k}\right) \in\left(A \cup A^{-1}\right)^{+},
$$

we let

$$
w^{-1} \equiv\left({ }^{g^{-1}} e_{k}, g_{k}^{-1}\right) \ldots\left({ }^{g_{1}{ }^{-1}} e_{1}, g_{1}^{-1}\right)
$$

and we let

$$
\Sigma(w) \equiv e_{1} \wedge{ }^{g_{1}} e_{2} \wedge \cdots \wedge \wedge^{g_{1} \ldots g_{k-1}} e_{k} \quad \text { and } \quad \Gamma(w) \equiv g_{1} \ldots g_{k} .
$$


Note that the element of $S$ represented by $w$ is $(\Sigma(w), \Gamma(w))$. Clearly, if $w \equiv u v$, then $\Sigma(w)=\Sigma(u) \wedge^{\Gamma(u)} \Sigma(v)$. The following lemma is now immediate.

LEMMA 3.3. Let $(Y, \wedge)$ be a semilattice and let $G$ be a group acting on $Y$. Assume that the semidirect product $S=Y \rtimes G$ is generated by A. Then for any $w \in F S I(A)$, $\Sigma\left(w w^{-1}\right)=\Sigma(w)$ holds in $Y$.

We know by Proposition 2.3 that if $Y \rtimes G$ is finitely generated, then $Y$ satisfies the maximum condition. Let $Y_{m}$ denote the set of maximal elements of $Y$. As before, let $A$ denote a finite generating set for $S$. Let $X=\{g \in G \mid(e, g) \in A$ for some $e \in Y\}$ and $Y_{0}=\left\{e \in Y \mid(e, g) \in A \cup A^{-1}\right.$ for some $\left.g \in X \cup X^{-1}\right\}$. Without loss of generality we may assume that $\left(Y_{0} \times 1\right) \cup\left(Y_{m} \times X\right) \subseteq A$. Define the following map

$$
\begin{aligned}
\mathbf{n}: & Y_{m} \times\left(X \cup X^{-1}\right)^{+} \rightarrow\left(A \cup A^{-1}\right)^{+} ; \\
\left(f_{1}, g_{1} g_{2} \ldots g_{k}\right) & \mapsto\left(f_{1}, g_{1}\right)\left(f_{2}, g_{2}\right) \ldots\left(f_{k}, g_{k}\right),
\end{aligned}
$$

where ${ }^{g_{j}} f_{j+1}=f_{j}(1 \leq j<k)$. By Lemma 2.1, $f_{j} \in Y_{m}$ for all $1 \leq j \leq k$. Note that for all $1 \leq j \leq k-1, f_{1}={ }^{g_{1} \ldots g_{j}} f_{j+1}$ holds, and thus the word $\mathbf{n}\left(f_{1}, g_{1} g_{2} \ldots g_{k}\right)$ represents $\left(f_{1}, g_{1} g_{2} \ldots g_{k}\right)$ in $S$. Throughout the paper, if $u \equiv \mathbf{n}\left(e, g_{1} \ldots g_{k}\right)$, then $u^{-1}$ will denote the word $\mathbf{n}\left(\tilde{e}, g_{k}^{-1} \ldots g_{1}^{-1}\right)$ where $e=g_{1} \ldots g_{k} \tilde{e}$. It is easy to see that $u^{-1}$ is indeed the inverse of $u$ in $S$.

Let $\alpha \in\left(X \cup X^{-1}\right)^{+}$and let

$$
c(\alpha, l)=\left\{u \cdot(l, 1) \cdot u^{-1} \mid u \equiv \mathbf{n}(e, \alpha), e \in Y_{m}, l \in Y_{0},{ }^{\alpha} l \leq e\right\} .
$$

The elements of $c(\alpha, l)$ are idempotents, in fact they represent $\left({ }^{\alpha} l, 1\right)$ in $S$. Clearly, if $Y$ satisfies the maximum condition, then $c(\alpha, l)$ is a finite set for all $l \in Y_{0}$ and $\alpha \in\left(X \cup X^{-1}\right)^{+}$.

\section{Main theorem, Part 1}

We prove the direct part of Theorem 3.2. The converse part is proved in the next section. Throughout we are going to work with the notation introduced in the previous section.

Assume that $S$ is given by the finite inverse semigroup presentation Inv $\langle A \mid P\rangle$. Without loss of generality we may assume that $\left(Y_{0} \times 1\right) \cup\left(Y_{m} \times X\right) \subseteq A$. Let $\Gamma(P)=$ $\{\Gamma(r)=\Gamma(p) \mid(r=p) \in P\}$. We show that $G=\operatorname{Grp}\langle X \mid \Gamma(P)\rangle$. We already know by Proposition 2.3 that $G$ is generated by the finite set $X$. Clearly, relations in $\Gamma(P)$ hold in $G$. We verify that any relation that holds in $G$ is a consequence of relations in $\Gamma(P)$. For this, assume that the relation $g_{1} \ldots g_{m}=h_{1} \ldots h_{k},\left(g_{i}, h_{j} \in X \cup X^{-1}\right)$ holds in $G$. Let $f \in Y_{m}$. Then $\mathbf{n}\left(f, g_{1} g_{2} \ldots g_{m}\right)=\mathbf{n}\left(f, h_{1} h_{2} \ldots h_{k}\right)$ holds in $S$. Thus, there exists a finite sequence of words

$$
\mathbf{n}\left(f, g_{1} \ldots g_{m}\right) \equiv q_{0}, q_{1}, \ldots, q_{t} \equiv \mathbf{n}\left(f, h_{1} \ldots h_{k}\right)
$$


where $q_{j+1}$ is obtained from $q_{j}$ by applying a relation in $P$ or a standard inverse semigroup relation. If $q_{j+1}$ is obtained from $q_{j}$ by applying a relation in $P$, then $\Gamma\left(q_{j+1}\right)$ is obtained from $\Gamma\left(q_{j}\right)$ by applying a relation in $\Gamma(P)$. If $q_{j+1}$ is obtained from $q_{j}$ by applying a standard inverse semigroup relation, then $\Gamma\left(q_{j+1}\right)$ is obtained from $\Gamma\left(q_{j}\right)$ by applying a sequence of standard group relations. It follows that there exists a finite sequence of words $g_{1} \ldots g_{m} \equiv \alpha_{0}, \alpha_{1}, \ldots, \alpha_{l} \equiv h_{1} \ldots h_{k}$, such that $\alpha_{j+1}$ is obtained from $\alpha_{j}$ by applying a relation in $\Gamma(P)$ or a standard group relation, verifying that $G=\operatorname{Grp}\langle X \mid \Gamma(P)\rangle$.

Next, we claim that $Y$ is finitely presented as an inverse semigroup with respect to the action of $G$. We already know that $Y$ satisfies the maximum condition and that $Y=\left\langle{ }^{G} Y_{0}\right\rangle$. On $\operatorname{FSI}\left({ }^{G} Y_{0}\right)$ we define the following set of relations:

$$
Q=\{\Sigma(r)=\Sigma(p) \mid(r=p) \in P\} \cup\left\{e=f \wedge e \mid e, f \in Y_{0}, e \leq f\right\} .
$$

We show that $Y=\operatorname{InvAct}_{G}\left\langle Y_{0} \mid Q\right\rangle$. It is clear that every relation of $Q$ and hence of $Q_{A}$ holds in $Y$. Assume that ${ }^{g_{1}} e_{1} \wedge{ }^{g_{2}} e_{2} \wedge \cdots \wedge{ }^{g_{m}} e_{m}={ }^{h_{1}} f_{1} \wedge{ }^{h_{2}} f_{2} \wedge \cdots \wedge{ }^{h_{k}} f_{k}$ holds in $Y$, where $e_{i}, f_{j} \in Y_{0}$ and $g_{i}, h_{j} \in G$. For all $1 \leq i \leq m, 1 \leq j \leq k$, we fix words $\alpha_{i}, \beta_{j} \in\left(X \cup X^{-1}\right)^{+}$so that $\alpha_{i}=g_{i}$ and $\beta_{j}=h_{j}$. Let $c_{i} \in c\left(\alpha_{i}, e_{i}\right)$ and $d_{j} \in c\left(\beta_{j}, f_{j}\right)$. Let $w_{1} \equiv c_{1} \ldots c_{m}$ and $w_{2} \equiv d_{1} \ldots d_{k}$. Clearly, the relations

$$
\Sigma\left(w_{1}\right)={ }^{g_{1}} e_{1} \wedge{ }^{g_{2}} e_{2} \wedge \cdots \wedge{ }^{g_{m}} e_{m} \quad \text { and } \quad \Sigma\left(w_{2}\right)={ }^{h_{1}} f_{1} \wedge{ }^{h_{2}} f_{2} \wedge \cdots \wedge{ }^{h_{k}} f_{k}
$$

are a consequence of relations in $\Re$.

We now verify that $\Sigma\left(w_{1}\right)=\Sigma\left(w_{2}\right)$ is a consequence of relations in $Q_{A}$. Since ${ }^{g_{1}} e_{1} \wedge \cdots \wedge{ }^{g_{m}} e_{m}={ }^{h_{1}} f_{1} \wedge \cdots \wedge{ }^{h_{k}} f_{k}$ holds in $Y$, the relation $w_{1}=w_{2}$ holds in $S$. Hence, there exists a finite sequence of words $w_{1} \equiv q_{0}, q_{1}, \ldots, q_{t} \equiv w_{2}$ such that $q_{j+1}$ is obtained from $q_{j}$ by applying a relation in $P$ or a standard inverse semigroup relation. We consider the following three cases.

(1) If $q_{j+1}$ is obtained from $q_{j}$ by applying a relation in $P$, then we may write $q_{j} \equiv t_{1} s t_{2}, q_{j+1} \equiv t_{1} z t_{2}$, where $(s=z) \in P$. It follows that $(\Sigma(s)=\Sigma(z)) \in \Sigma(P)$ and $(\Gamma(s)=\Gamma(z)) \in \Gamma(P)$. In particular, $\Gamma\left(t_{1} s\right)=\Gamma\left(t_{1} z\right)$ holds in $G$ and we obtain that

$$
\begin{aligned}
\Sigma\left(q_{j}\right) & \equiv \Sigma\left(t_{1}\right) \wedge^{\Gamma\left(t_{1}\right)} \Sigma(s) \wedge^{\Gamma\left(t_{1} s\right)} \Sigma\left(t_{2}\right) \\
& =\Sigma\left(t_{1}\right) \wedge^{\Gamma\left(t_{1}\right)} \Sigma(z) \wedge^{\Gamma\left(t_{1} s\right)} \Sigma\left(t_{2}\right) \\
& =\Sigma\left(t_{1}\right) \wedge^{\Gamma\left(t_{1}\right)} \Sigma(z) \wedge^{\Gamma\left(t_{1} z\right)} \Sigma\left(t_{2}\right) \equiv \Sigma\left(q_{j+1}\right)
\end{aligned}
$$

verifying that $\Sigma\left(q_{j+1}\right)$ is obtained from $\Sigma\left(q_{j}\right)$ by applying relations in $Q_{A}$.

(2) If $q_{j+1}$ is obtained from $q_{j}$ by applying a relation of the form $w w^{-1} w=w$, then we may write $q_{j} \equiv t_{1} w w^{-1} w t_{2}, q_{j+1} \equiv t_{1} w t_{2}$. Let $u \equiv w w^{-1}$, and $v \equiv w w^{-1} w$. By Lemma 3.3 we have that $\Sigma(u)=\Sigma(w)$. Clearly $\Gamma(v)=\Gamma(w)$ holds in $G$ and we 
have that

$$
\begin{aligned}
\Sigma\left(q_{j}\right) & \equiv \Sigma\left(t_{1}\right) \wedge \Gamma\left(t_{1}\right) \Sigma(u) \wedge \Gamma\left(t_{1} u\right) \Sigma(w) \wedge^{\Gamma\left(t_{1} v\right)} \Sigma\left(t_{2}\right) & & \\
& =\Sigma\left(t_{1}\right) \wedge \Gamma{ }^{\left(t_{1}\right)} \Sigma(w) \wedge \Gamma\left(t_{1} u\right) \Sigma(w) \wedge^{\Gamma\left(t_{1} v\right)} \Sigma\left(t_{2}\right) & & \text { by } Q_{A} \\
& =\Sigma\left(t_{1}\right) \wedge \Gamma{ }^{\left(t_{1}\right)} \Sigma(w) \wedge \Gamma\left(t_{1}\right) \Sigma(w) \wedge^{\left(t_{1} v\right)} \Sigma\left(t_{2}\right) & & \text { since } \Gamma\left(t_{1} u\right)=\Gamma\left(t_{1}\right) \\
& =\Sigma\left(t_{1}\right) \wedge \Gamma{ }^{\left(t_{1}\right)} \Sigma(w) \wedge \Gamma\left(t_{1} v\right) \Sigma\left(t_{2}\right) & & \text { by } Q_{A} \\
& =\Sigma\left(t_{1}\right) \wedge^{\left(t_{1}\right)} \Sigma(w) \wedge^{\Gamma\left(t_{1} w\right)} \Sigma\left(t_{2}\right) & & \text { since } \Gamma(w)=\Gamma(v) \\
& \equiv \Sigma\left(q_{j+1}\right) & &
\end{aligned}
$$

proving that $\Sigma\left(q_{j}\right)=\Sigma\left(q_{j+1}\right)$ is a consequence of relations in $Q_{A}$.

(3) If $q_{j+1}$ is obtained from $q_{j}$ by applying a relation of the form $w_{1} w_{1}^{-1} w_{2} w_{2}^{-1}=w_{2} w_{2}^{-1} w_{1} w_{1}^{-1}$, then we may write $q_{j} \equiv t_{1} w_{1} w_{1}^{-1} w_{2} w_{2}^{-1} t_{2}$ and $q_{j+1} \equiv t_{1} w_{2} w_{2}^{-1} w_{1} w_{1}^{-1} t_{2}$. Let $u \equiv w_{1} w_{1}^{-1}, v \equiv w_{2} w_{2}^{-1}$. Then

$$
\begin{aligned}
& \Sigma\left(q_{j}\right) \equiv \Sigma\left(t_{1}\right) \wedge^{\Gamma\left(t_{1}\right)} \Sigma(u) \wedge^{\Gamma\left(t_{1} u\right)} \Sigma(v) \wedge^{\Gamma\left(t_{1} u v\right)} \Sigma\left(t_{2}\right) \\
& =\Sigma\left(t_{1}\right) \wedge^{\Gamma\left(t_{1}\right)} \Sigma(u) \wedge^{\Gamma\left(t_{1}\right)} \Sigma(v) \wedge^{\Gamma\left(t_{1}\right)} \Sigma\left(t_{2}\right) \quad \text { since } \Gamma(u)=\Gamma(u v)=1 \\
& =\Sigma\left(t_{1}\right) \wedge \Gamma\left(t_{1}\right) \Sigma(v) \wedge^{\Gamma\left(t_{1}\right)} \Sigma(u) \wedge \Gamma\left(t_{1}\right) \Sigma\left(t_{2}\right) \quad \text { by } \Re \\
& =\Sigma\left(t_{1}\right) \wedge^{\Gamma\left(t_{1}\right)} \Sigma(v) \wedge^{\Gamma\left(t_{1} v\right)} \Sigma(u) \wedge^{\Gamma\left(t_{1} v u\right)} \Sigma\left(t_{2}\right) \quad \text { since } \Gamma(v)=\Gamma(v u)=1 \\
& \equiv \Sigma\left(q_{j+1}\right)
\end{aligned}
$$

proving that $\Sigma\left(q_{j}\right)=\Sigma\left(q_{j+1}\right)$ is a consequence of relations in $Q_{A}$.

It follows that there exists a finite sequence of words $\Sigma\left(w_{1}\right) \equiv u_{0}, u_{1}, \ldots, u_{l} \equiv$ $\Sigma\left(w_{2}\right)$ such that $u_{j+1}$ is obtained from $u_{j}$ by applying a relation of $Q_{A}$ and we may deduce that $Y=\operatorname{InvAct}_{G}\left\langle Y_{0} \mid Q\right\rangle$.

\section{Main theorem, Part 2}

In this section we prove the converse part of our main theorem. Let $G=\operatorname{Grp}\langle X \mid P\rangle$, where $X$ is a finite set and $P$ is a finite set of relations. Without loss of generality we may assume that for all $g \in X$ the relations $g g^{-1}=g^{-1} g=1$ and $g=g 1=1 g$ are contained in $P$. Let $Y$ be a semilattice satisfying the maximum condition and let $Y_{m}$ denote the set of maximal elements of $Y$. Assume that $Y=\operatorname{InvAct}_{G}\left\langle Y_{0} \mid Q\right\rangle$, where $Y_{0}$ is a finite subset of $Y$ and $Q$ is a finite set of relations on $\operatorname{FSI}\left({ }^{G} Y_{0}\right)$. By Proposition 3.1 we may assume that $Y_{m} \subseteq Y_{0}$. Consider the semidirect product $S=Y \rtimes G$. We showed in Proposition 2.3 that $S$ is finitely generated as an inverse semigroup by $A=\left(Y_{0} \times\{1\}\right) \cup\left(Y_{m} \times X\right)$. Clearly $A^{-1}=\left(Y_{0} \times\{1\}\right) \cup\left(Y_{m} \times X^{-1}\right)$. Define the following relations on $\left(A \cup A^{-1}\right)^{+}$:

(R1) $(e, 1)(f, h)=(f, 1)(e, h)$, where $e, f \in Y_{m}$;

(R2) $(e, g)(f, h)=(l, 1)(e, g)(f, h)$, where $e, f, l \in Y_{m}$ and $l={ }^{g} f$;

(R3) $(e, 1)(f, h)=(e, 1)(f, h)(l, 1)$, where $e, f, l \in Y_{m}$ and $e={ }^{h} l$. 
We let $R$ denote the set of these relations. Let

$$
R_{G}=\left\{\mathbf{n}(e, p)=\mathbf{n}(e, q) \mid e \in Y_{m},(p=q) \in P\right\}
$$

Since $Y_{m}$ and $P$ are finite sets, we have that $R_{G}$ is a finite set of relations. For every relation $p \equiv{ }^{g_{1}} e_{1} \wedge \cdots \wedge{ }^{g_{m}} e_{m}={ }^{h_{1}} f_{1} \wedge \cdots \wedge{ }^{h_{k}} f_{k} \equiv q$ in $Q$ we fix words $\alpha_{i}, \beta_{j}$ over $X \cup X^{-1}$ such that $\alpha_{i}=g_{i}$ and $\beta_{j}=h_{j}$. Define the following set of relations

$$
R_{p, q}=\left\{c_{11} \ldots c_{1 m}=c_{21} \ldots c_{2 k} \mid c_{1 j} \in c\left(\alpha_{j}, e_{j}\right), c_{2 j} \in c\left(\beta_{j}, f_{j}\right)\right\}
$$

and let

$$
R_{Y}=\bigcup_{((p=q) \in Q)} R_{p, q} \cup\left\{(e, g)=(f, 1)(e, g) \mid e \leq f, e, f \in Y_{0}\right\}
$$

Since $Y$ satisfies the maximum condition, the sets $c\left(\alpha_{j}, e_{j}\right)$ and $c\left(\beta_{j}, f_{j}\right)$ are finite and hence for all $(p=q) \in Q$ the set $R_{p, q}$ is finite. It follows that $R_{Y}$ is finite as well.

Let $\mathbf{R}=R \cup R_{G} \cup R_{Y}$. Our aim is to show that $S=\operatorname{Inv}\langle A \mid \mathbf{R}\rangle$. Clearly $\mathbf{R}$ is a finite set of relations and all relations in $\mathbf{R}$ hold in $S$. According to Proposition 1.1 we need to verify that any relation $w_{1}=w_{2}$ that holds in $S$ is a consequence of relations in $\mathbf{R}$ and of standard inverse semigroup relations. The following key proposition establishes a way of rewriting an arbitrary word over $A \cup A^{-1}$ into a word of special form.

Proposition 5.1. Let $w \equiv\left(e_{1}, g_{1}\right) \ldots\left(e_{k-1}, g_{k-1}\right)\left(e_{k}, g_{k}\right) \in\left(A \cup A^{-1}\right)^{+}$and let $c_{1}=\left(e_{1}, 1\right)$. Then, the relation $w=c_{1} c_{2} \ldots c_{k} u$ where $u \equiv \mathbf{n}\left(f, g_{1} \ldots g_{k}\right)$ for some $f \in Y_{m}$ and $c_{j} \in c\left(g_{1} \ldots g_{j-1}, e_{j}\right)$ for all $(2 \leq j \leq k)$ is a consequence of relations in R. Moreover, $c_{k}(f, 1)=c_{k}$ also holds in $S$.

We need the following lemma to prove Proposition 5.1.

LEMMA 5.2. Let $w \equiv \mathbf{n}\left(e, g_{1} g_{2} \ldots g_{k}\right)\left(f, g_{k+1}\right)$, where $f \in Y_{m}$. Then there exists $l \in Y_{m}$ such that the relation $w=(e, 1) \mathbf{n}\left(l, g_{1} g_{2} \ldots g_{k+1}\right)$ is a consequence of relations in $\mathbf{R}$. In particular, $l={ }^{g_{1} \ldots g_{k}} f$.

PROOF. Let $l_{k}={ }^{g_{k}} f$. Since $f \in Y_{m}$, we have that $l_{k} \in Y_{m}$. Let $l_{1}, l_{2}, \ldots, l_{k-1} \in Y_{m}$ such that $l_{j}={ }^{g} l_{j+1}$ holds. Such elements exist by Lemma 2.1. Note that ${ }^{g_{1} \ldots g_{k}} f=l_{1}$. Assume that $\mathbf{n}\left(e, g_{1} \ldots g_{k}\right) \equiv\left(e, g_{1}\right) \ldots\left(e_{k}, g_{k}\right)$. Then

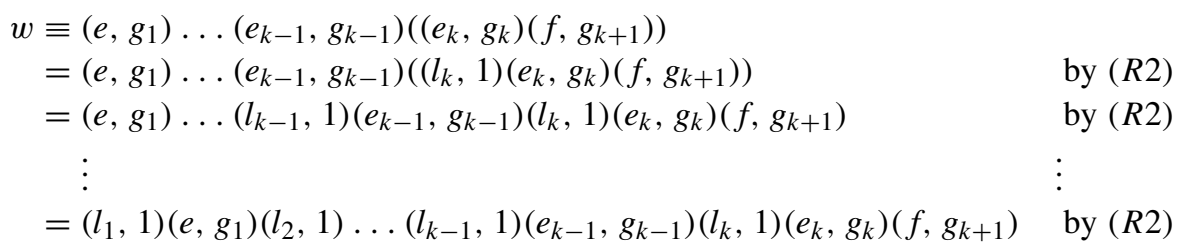




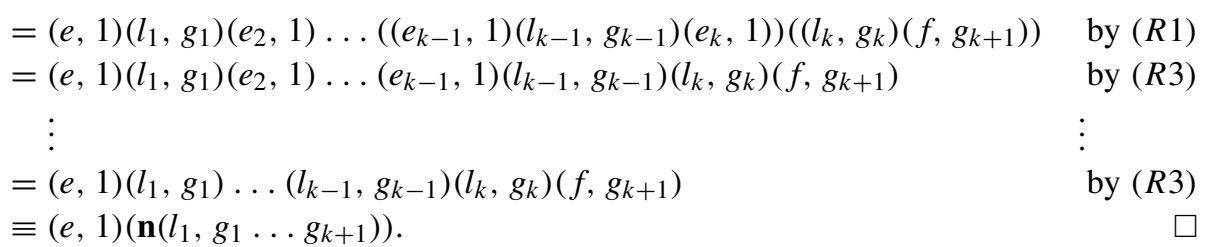

Similarly we can prove the following result.

LEMMA 5.3. Let $f \in Y_{m}$ and consider $w \equiv(f, 1) \mathbf{n}\left(e, g_{1} g_{2} \ldots g_{k}\right)$. Then $w=$ $(e, 1) \mathbf{n}\left(f, g_{1} g_{2} \ldots g_{k}\right)$ is a consequence of relations in $R$.

Proof of PROPOSITION 5.1. We proceed by induction on the length of $w$. It is easy to verify that the proposition holds for words of length one. Assume that the proposition is true for all words whose length is less then $k$.

Let $w \equiv\left(e_{1}, g_{1}\right) \ldots\left(e_{k-1}, g_{k-1}\right)\left(e_{k}, g_{k}\right)$. Applying the inductive hypothesis we obtain that $w=c_{1} c_{2} \ldots c_{k-1} u\left(e_{k}, g_{k}\right)$, where $c_{1}=\left(e_{1}, 1\right), c_{j} \in c\left(g_{1} \ldots g_{j-1}, e_{j}\right)$, $u \equiv \mathbf{n}\left(f, g_{1} \ldots g_{k-1}\right)$ for some $f \in Y_{m}$ and $c_{k-1}(f, 1)=c_{k-1}$. Let $e \in Y_{m}$ such that $e_{k} \leq e$. By Lemma 5.2, we have that $u(e, 1)=(f, 1) \mathbf{n}\left(l, g_{1} \ldots g_{k-1}\right)$ where $l={ }^{g_{1} \ldots g_{k-1}} e$. We let $v \equiv \mathbf{n}\left(l, g_{1} \ldots g_{k-1}\right)$. Then

$$
\begin{aligned}
w & =c_{1} c_{2} \ldots c_{k-1} u\left(e_{k}, g_{k}\right) & & \\
& =c_{1} c_{2} \ldots c_{k-1} u(e, 1)\left(e_{k}, g_{k}\right) & & \text { by } R_{Y} \\
& =c_{1} c_{2} \ldots c_{k-1}(f, 1) v\left(e_{k}, g_{k}\right) & & \text { by Lemma } 5.2 \\
& =c_{1} c_{2} \ldots c_{k-1} v\left(e_{k}, g_{k}\right) & & \text { since } c_{k-1}(f, 1)=c_{k-1} \\
& =c_{1} c_{2} \ldots c_{k-1} v\left(e_{k}, 1\right)\left(e_{k}, g_{k}\right) & & \text { by } R_{Y} \\
& =c_{1} c_{2} \ldots c_{k-1} v\left(e_{k}, 1\right) v^{-1} v\left(e_{k}, g_{k}\right) & & \text { by } \Re .
\end{aligned}
$$

Since $l={ }^{g_{1} \ldots g_{k-1}} e$ and $e_{k} \leq e$ we have that $c_{k} \equiv v\left(e_{k}, 1\right) v^{-1} \in c\left(g_{1} \ldots g_{k-1}, e_{k}\right)$. Moreover, by applying a relation from $R_{G}$, we obtain that $c_{k}(l, 1)=c_{k}$. If $e_{k} \in Y_{m}$, that is, if $e=e_{k}$, then we also obtain that $v\left(e_{k}, g_{k}\right) \equiv \mathbf{n}\left(l, g_{1} \ldots g_{k}\right)$. If $e_{k} \in Y_{0} \backslash Y_{m}$, then by the above we have $w=c_{1} c_{2} \ldots c_{k-1} v\left(e_{k}, 1\right)$ and so by applying relations in $R_{Y}$ and standard inverse semigroup relations we obtain that

$$
w=c_{1} c_{2} \ldots c_{k-1} v\left(e_{k}, 1\right) v^{-1} v(e, 1) .
$$

Since $l={ }^{g_{1} \ldots g_{k-1}} e$ we have that $v(e, 1) \equiv \mathbf{n}\left(l, g_{1} \ldots g_{k-1} 1\right)$ holds. It now follows that $w=c_{1} \ldots c_{k} \mathbf{n}\left(l, g_{1} \ldots g_{k}\right)$.

We now complete the proof of Theorem 3.2. Assume that

$$
w_{1} \equiv\left(e_{1}, g_{1}\right) \ldots\left(e_{m}, g_{m}\right)=\left(f_{1}, h_{1}\right) \ldots\left(f_{k}, h_{k}\right) \equiv w_{2} .
$$

holds in $S$. Let $c_{1}=\left(e_{1}, 1\right)$ and $d_{1}=\left(f_{1}, 1\right)$. By Proposition 5.1, we have that $w_{1}=c_{1} c_{2} \ldots c_{m} u$, where $c_{j} \in c\left(g_{1} \ldots g_{j-1}, e_{j}\right), u \equiv \mathbf{n}\left(e, g_{1} \ldots g_{m}\right)$ for some $e \in Y_{m}$ and $c_{m}(e, 1)=c_{m}$. Similarly $w_{2}=d_{1} d_{2} \ldots d_{k} v$, where $d_{j} \in c\left(h_{1} \ldots\right.$ $\left.h_{j-1}, f_{j}\right), \quad v \equiv \mathbf{n}\left(f, h_{1} \ldots h_{k}\right)$ for some $f \in Y_{m}$ and $d_{k}(f, 1)=d_{k}$. We write 
$\left(w_{1}\right)_{Y} \equiv c_{1} c_{2} \ldots c_{m}$ and $\left(w_{2}\right)_{Y} \equiv d_{1} d_{2} \ldots d_{k}$. We prove in three steps that $w_{1}=w_{2}$ is a consequence of relations in $\mathbf{R}$ and of standard inverse semigroup relations.

STEP 1. Since $w_{1}=w_{2}$ in $S$, we have that $\Gamma\left(w_{1}\right)=\Gamma\left(w_{2}\right)$ holds in $G$. Hence, there exists a finite sequence of words $\Gamma\left(w_{1}\right) \equiv \alpha_{0}, \alpha_{1}, \ldots, \alpha_{t} \equiv \Gamma\left(w_{2}\right)$ such that $\alpha_{j+1}$ is obtained from $\alpha_{j}$ by applying a relation in $P$ or a standard group relation. It follows that there exists a finite sequence of words $\mathbf{n}\left(e, g_{1} \ldots g_{m}\right) \equiv u_{0}, \ldots, u_{t}$ $\equiv \mathbf{n}\left(e, h_{1} \ldots h_{k}\right)$ such that $\beta_{j+1}$ is obtained from $\beta_{j}$ by applying a relation in $R_{G}$. It follows that $\left(w_{1}\right)_{Y} u=\left(w_{1}\right)_{Y} u_{t}$ holds in $S$.

STEP 2. Since $w_{1}=w_{2}$ in $S$, we have that $\Sigma\left(w_{1}\right)=\Sigma\left(w_{2}\right)$ holds in $Y$, and hence there exists a finite sequence of words $\Sigma\left(w_{1}\right) \equiv \gamma_{1}, \gamma_{2}, \ldots, \gamma_{q} \equiv \Sigma\left(w_{2}\right)$ such that $\gamma_{j+1}$ is obtained from $\gamma_{j}$ by applying a relation in $Q_{A}$ or a standard inverse semigroup relation. Now we construct a finite sequence of words $\left(w_{1}\right)_{Y} \equiv \delta_{1}, \delta_{2}, \ldots, \delta_{q} \equiv\left(w_{2}\right)_{Y}$ in the following way. If $\gamma_{j+1}$ is obtained from $\gamma_{j}$ by applying a relation $p=q$ in $Q$, then $\delta_{j+1}$ is obtained from $\delta_{j}$ by applying a relation in $R_{p, q}$. If $\gamma_{j+1}$ is obtained from $\gamma_{j}$ by applying a relation ${ }^{g} p={ }^{g} q$ in $Q_{A}$, then $\delta_{j+1}$ is obtained from $\delta_{j}$ by applying a relation in $R_{p, q}$ and relations in $R_{G}$. If $\gamma_{j+1}$ is obtained from $\gamma_{j}$ by applying inverse semigroup relations then $\delta_{j+1}$ is obtained from $\delta_{j}$ by applying inverse semigroup relations. Thus, we obtain that $\left(w_{1}\right)_{Y} u_{t}=\left(w_{2}\right)_{Y} u_{t}$ holds in $S$.

STEP 3. Finally we show that $\left(w_{2}\right)_{Y} u_{t}=\left(w_{2}\right)_{Y} v$ holds. Since $d_{k}=d_{k}(f, 1)$, we have that $\left(w_{2}\right)_{Y}=\left(w_{2}\right)_{Y}(f, 1)$. It follows that

$$
\begin{aligned}
\left(w_{2}\right)_{Y} u_{t} & \equiv\left(w_{2}\right)_{Y}\left(\mathbf{n}\left(e, h_{1} \ldots h_{k}\right)\right) \\
& =\left(w_{2}\right)_{Y}(f, 1)\left(\mathbf{n}\left(e, h_{1} \ldots h_{k}\right)\right) \\
& =\left(w_{2}\right)_{Y}(e, 1)\left(\mathbf{n}\left(f, h_{1} \ldots h_{k}\right)\right) \quad \text { by Lemma } 5.3 .
\end{aligned}
$$

Finally we claim that $\left(w_{2}\right)_{Y}(e, 1)=\left(w_{2}\right)_{Y}$. Since $w_{1}=c_{1} \ldots c_{m} \mathbf{n}\left(e, g_{1} \ldots g_{m}\right)$, we have that $\Sigma\left(w_{1}\right)=\Sigma\left(\left(w_{1}\right)_{Y}\right) \wedge e$. On the other hand $\Sigma\left(w_{1}\right)=\Sigma\left(w_{2}\right)$ holds. It follows that $\Sigma\left(w_{2}\right) \wedge e=\Sigma\left(w_{2}\right)$ holds in $Y$. Thus, there exists a finite sequence of words $\left(w_{2}\right)_{Y}(e, 1) \equiv \zeta_{1}, \ldots, \zeta_{l}=\left(w_{2}\right)_{Y}$, such that $\zeta_{j+1}$ is obtained from $\zeta_{j}$ using relations in $R_{Y} \cup R_{G}$ and standard inverse semigroup relations. We may now deduce that $w_{1}=w_{2}$ is indeed a consequence of relations in $\mathbf{R}$ and of inverse semigroup relations.

\section{Examples}

To illustrate our results, we give two examples. In both examples the infinite antichain $A=\left\{\ldots e_{-1}, e_{0}, e_{1}, \ldots\right\}$ plays a central role.

EXAmple 6.1. Let $Y$ denote the free semilattice generated by infinitely many elements $A=\left\{\ldots e_{-1}, e_{0}, e_{1}, \ldots\right\}$ with an identity element $1_{Y}$ adjoined. Let $G=\langle g\rangle$ be the infinite cyclic group. For each $n \in \mathbb{Z}$ define $\psi_{g^{n}}: e_{i} \mapsto e_{i+n}, 1_{Y} \mapsto 1_{Y}$ and extend this map to an automorphism of $Y$. It is clear that an action of $G$ on $Y$ 
is defined. Since $Y$ is the free semilattice with an identity adjoined and since $e_{n}=g^{n} e_{0}$, we have that $Y$ is generated by $Y_{0}=\left\{e_{0}, 1_{Y}\right\}$ with respect to this action. It is immediate that $Y$ satisfies the maximum condition. We show that $S=Y \rtimes G$ is finitely presented by showing that $Y$ is finitely presented with respect to the action of $G$. Let $Q=\left\{e_{0} \wedge e_{0}=e_{0}, 1_{Y} \wedge 1_{Y}=1_{Y}\right\}$. We show that $Y=\operatorname{InvAct}_{G}\left\langle Y_{0} \mid Q\right\rangle$. Assume that the relation $p=q$ holds in $Y$. Since $Y$ is the free semilattice with an identity element adjoined, we have that ${ }^{n} e_{0}$ appears in the word $p$ if and only if it appears in $q$. Thus, we can construct a finite sequence of words $p \equiv u_{0}, \ldots, u_{n} \equiv q$ such that $u_{j+1}$ is obtained from $u_{j}$ by applying relations in $Q_{A}$ or standard inverse semigroup relations.

EXAMPLE 6.2. Let $A$ be the infinite antichain and adjoin an identity element $1_{Y}$ on the top and a zero element $0_{Y}$ on the bottom. Denote the semilattice obtained by $Y$. Let $G=\langle g\rangle$ be the infinite cyclic group. For each $n \in \mathbb{Z}$ define $\psi_{g^{n}}: e_{i} \mapsto e_{i+n}$, $1_{Y} \mapsto 1_{Y}, 0_{Y} \mapsto 0_{Y}$. It is clear that an action of $G$ on $Y$ is defined. Note that $Y$ is generated by $Y_{0}=\left\{e_{0}, 1_{Y}\right\}$ with respect to this action. Indeed $e_{n}={ }^{g^{n}} e_{0}$ and $e_{n} \wedge e_{m}=0_{Y}$ for all $n \neq m$. We show that $S=Y \rtimes G$ is not finitely presented by showing that $Y$ is not finitely presented with respect to the action of $G$.

Assume that $Y$ is finitely presented with respect to the action of $G$. By Proposition 3.1 we may assume that $Y=\operatorname{InvAct}_{G}\left\langle Y_{0} \mid Q\right\rangle$, where $Q$ is a finite set of relations on $\operatorname{FSI}\left({ }^{G} Y_{0}\right)$. Consider an arbitrary word

$$
w \equiv g^{n_{1}} e_{0} \wedge \cdots \wedge{ }^{g^{n_{i}}} e_{0} \wedge \cdots \wedge{ }^{g^{n_{j}}} e_{0} \ldots \wedge{ }^{g^{n_{m}}} e_{0} .
$$

Assume that $n_{i} \leq n_{k} \leq n_{j}$ for all $1 \leq k \leq m$ and let $d(w)=n_{j}-n_{i}$. We call $d(w)$ the distance in $w$. Let $d \in \mathbb{N}$ so that $d=d(u)$ where $u=v$ or $v=u$ is a relation in $Q$ and if $w$ is word appearing on one side of a relation in $Q$ then $d(w) \leq d$. Owing to the defined action of $G$ on $Y$ we also have that for all relations $u=v$ in $Q_{A}, d(u), d(v) \leq d$.

Clearly the relation $p \equiv g^{d+1} e_{0} \wedge e_{0}={ }^{g d+2} e_{0} \wedge e_{0} \equiv q$ holds in $Y$ and $d<d(p)<$ $d(q)$. If $p=q$ is a consequence of relations in $Q_{A}$ and of inverse semigroup relations, then there exists a finite sequence of words ${ }^{d+1} e_{0} \wedge e_{0} \equiv u_{0}, u_{1} \ldots u_{t} \equiv g^{d+2} e_{0} \wedge e_{0}$ so that $u_{j+1}$ is obtained from $u_{j}$ by applying relations in $Q_{A}$ and of standard inverse semigroup relations. Since $d<d(p)$ there exists no relation in $Q_{A}$ that can be applied to $p$. On the other hand, by applying inverse semigroup relations the distance in $p$ cannot be increased. It follows that $p=q$ is not a consequence of relations in $Q_{A}$ and of standard inverse semigroup relations and, hence, $S$ is not finitely presented.

\section{References}

[1] I. M. Araújo and N. Ruškuc, 'On finite presentability of direct products of semigroups', Algebra Colloq. 7 (2000), 83-91.

[2] E. R. Dombi, 'Automatic $S$-acts and inverse semigroup presentations', PhD Thesis, University of St Andrews, St Andrews, Scotland, UK, 2004.

[3] P. Gallagher and N. Ruškuc, 'On finite generations and presentability of Schützenberger products', J. Aust. Math. Soc. 83 (2007), 357-367.

[4] T. G. Lavers, 'Presentations of general products', J. Algebra 204 (1998), 733-741. 
[5] M. V. Lawson, 'Inverse semigroups', The Theory of Partial Symmetries (World Scientific, River Edge, NJ, 1998).

[6] E. F. Robertson, N. Ruškuc and M. R. Thomson, 'Finite generation and presentability of wreath products of monoids', J. Algebra 266 (2003), 382-392.

[7] E. F. Robertson, N. Ruškuc and J. Wiegold, 'Generators and relations of direct products of semigroups', Trans. Amer. Math. Soc. 350 (1998), 2665-2685.

E. R. DOMBI, School of Mathematics and Statistics, University of St Andrews, St Andrews KY16 9SS, Scotland, UK

e-mail: erzsi@mcs.st-and.ac.uk

N. RUŠKUC, School of Mathematics and Statistics, University of St Andrews, St Andrews KY16 9SS, Scotland, UK

e-mail: nik@mcs.st-and.ac.uk 